http://dx.doi.org/10.21611/qirt.2015.0121

\title{
Thermogram asymmetry analysis for the Evaluation of Mammary cancer in 7, 12-dimethyl benz (a)nthracene (DMBA) induced Wistar Rats
}

\author{
Angeline Kirubha. S. P $^{\text {* }}$, Anburajan. M ${ }^{1}$, Venkataraman. B ${ }^{2}$, Akila. $\mathbf{R}^{2}$, Sharath. $\mathbf{D}^{2}$, \\ Department of Biomedical Engineering, SRM University, Kattankulathur- 603 203, Tamilnadu, India ${ }^{1}$
}

Angeline.sp@ktr.srmuniv.ac.in,hod.biomedi@ktr.srmuniv.ac.in

IndiraGandhiCenter for Atomic Research, Kalpakkam, ${ }^{2}$

*- Corresponding Author

\section{Abstract}

Background: Thermography was widely used in diagnosis, prognosis of several diseases, and to study progress of treatment with animal model. Animal skin surface temperatures can be detected using Infra Red camera which is helpful for the assessment of physiological responses associated with the regulation of body temperature by asymmetrical analysis. Infrared imaging of animals has been used in the past to detect changes in skin surface temperature associated with cancer, rheumatoid arthritis, animals' temperature adaptation study and also in anesthesia study. Diagnosing breast cancer in early stage itself has a greater effect on the prognosis. The aim of this study was to evaluate the potential of thermography in an evaluation of breast cancer. Methods: In this present work whole body thermogram of six wistar rats, of chemical carcinogen 7, 12-dimethyl benz (a) nthracene (DMBA) induced in the lower right flank region were captured over a period of nine weeks . Asymmetrical analysis of temperature distribution in thermal images was done in comparison with histopathology results as gold standard and also with serum Carcino Embryonic Antigen (CEA) levels. Result: Temperature difference between 
the MC induced lower right flank region and lower left (control) flank region was Significant (with $\mathrm{p}$ value $<0.001$ ), whereas in the other regions (Abdomen and Shoulder) there was no significant difference in temperature between right and left sides. Percentage of asymmetrical temperature difference in the $\mathrm{MC}$ induced lower flank region was 0.5 to $2 \%$ whereas in the other regions it was $<0.5 \%$. Green pixel distribution in RGB color histogram was Asymmetrical in the MC induced lower flank region. Conclusion: Temperature reduction was observed in the $\mathrm{MC}$ induced region after seventh day of chemical carcinogen induction. Asymmetrical temperature distribution analysis may be one of the methods of finding any abnormalities. Thermography can be used for clinical diagnosis as well as for studying tumor development.

Key words -Infrared, DMBA-Chemical Carcinogen, Asymmetrical temperature Analysis, Color histogram

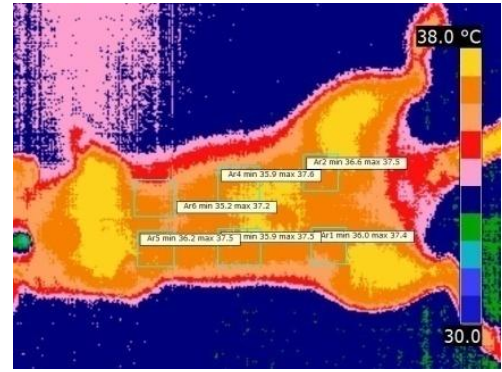

(A)

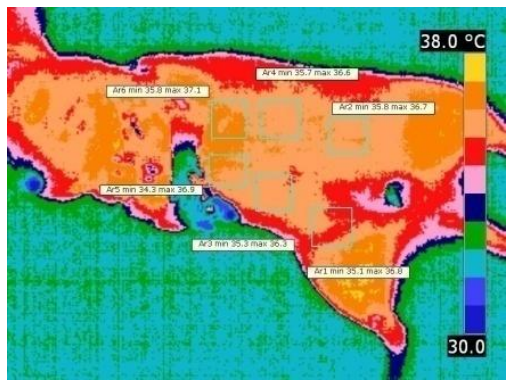

(C)

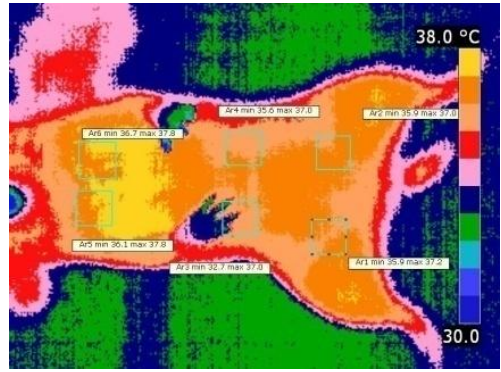

(B)

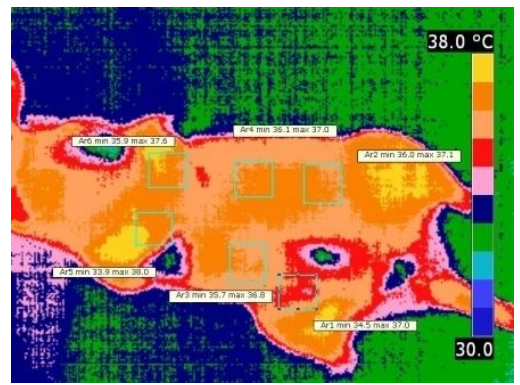

(D)

Figure. 1 Whole body digital infrared images of a single rat taken on different weeks (A. week1, B. week2, C. week3, D. week4) after tumor cancer induction by DMBA which showing the tumor growth by color change from thick orange (A), then light orange (B), then red $\operatorname{color}(C)$ and then to red color with pink center in the fourth week indicating a reduction in skin temperature progressively 